\title{
Prevalence and Antibiotic Resistant Pattern of Pseudomonas aeruginosa at a Tertiary Care Centre of North India
}

\author{
Trinain Kumar Chakraverti ${ }^{1}$ and Purti C. Tripathi $^{2 *}$ \\ ${ }^{1}$ Department of Microbiology, Patna Medical College, Patna-800004, India \\ ${ }^{2}$ Department of Microbiology, Government Medical College, Chhindwara, \\ Madhya Pradesh - 480001, India \\ *Corresponding author
}

\begin{abstract}
A B S T R A C T
Keywords

Pseudomonas

aeruginosa, Multi-drug

resistance, Extended

spectrum of $\beta$ lactamase

(ESBL), Metallo $\beta$

lactamase (MBL)

Article Info

Accepted:

08 August 2018

Available Online:

10 September 2018

The aim of this study was to analyze the extended spectrum of $\beta$ lactamase (ESBL), metallo $\beta$ lactamase (MBL) and AmpC production in Pseudomonas aeruginosa in various clinical samples. A Total of 100 clinical isolates of $P$. aeruginosa were collected from different clinical specimen and confirmed by standard tests. Antibiotic susceptibility was determined by the Kirby-Bauer disc diffusion method. ESBL screening was done using 3rd generation cephalosporins and confirmatory combined double disc test, imipenem-EDTA double disc synergy test for MBL enzyme and AmpC test using Cefoxitin disc. Out of 100 clinical P.aeruginosa isolates, 33\% were ESBL producer, $18 \%$ MBL producer both ESB and MBL 9\% and none were AmpC producer. Imipenem (81\%), meropenem (82\%), aminoglycosides (amikacin (72\%), tobramycin (74\%), netilmycin (71\%) and Polymyxin $\mathrm{B}(100 \%)$ and colistin $(100 \%)$ has got the better antipseudomonal activity. $28(28 \%)$ P.aeruginosa was found to be Multi Drug Resistant (MDR). This study highlights the prevalence of ESBL, MBL and MDR P.aeruginosa. In our study Carbapenems and aminoglycosides are promising drugs with antipseudomonal activity while polymyxin $b$ and colstin use as reserved drug.
\end{abstract}

\section{Introduction}

Pseudomonas aeruginosa belongs to a large group of aerobic, non-fermenting saprophytic, gram-negative bacilli widespread in nature, particularly in moist environment. (Govan, 2008; Du Bois et al., 2001) However, its profound ability to survive on inert materials, minimal nutritional requirement, tolerance to a wide variety of physical conditions and its relative resistance to several unrelated antimicrobial agents and antiseptics, contributes enormously to its ecological success and its role as an effective opportunistic pathogen. (Gales et al., 2001) Pseudomonas aeruginosa has emerged as a major cause of infection in the last few decades. It is an increasingly prevalent opportunistic pathogen and is the fourth most frequently isolated nosocomial pathogen accounting for $10 \%$ of all hospital acquired infections. (Pathi et al.,) The organism has been incriminated in cases of meningitis, septicemia, pneumonia, ocular and burn 
infection, osteomyelitis, cystic fibrosis related lung infection, malignant external otitis and urinary tract infections with colonized patients being an important reservoir (Hernandez et al., 1997) Pseudomonas aeruginosa shows innate resistance to many disinfectants and antibiotics. (Syed Arshi et al., 2007) Nosocomial infections mainly caused by ESBL, MBL, MDR and PDR P.aeruginosa strains creates enormous burden of morbidity, mortality and high health care cost.

The aims and objectives of this study is to determine the prevalence of (i) Pseudomonas aeruginosa strains from various clinical samples and their antibiotic resistance pattern. (ii) Prevalence of ESBL, MBL and AmpC production in Pseudomonas aeruginosa from various clinical samples in our tertiary care hospital PMCH Patna, Bihar, India.

\section{Materials and Methods}

The study was carried out in Department of Microbiology, Patna Medical College, Patna during the period from October 2017 till March 2018. All the samples were obtained from PMCH hospital, to Microbiology department were processed as per standard protocol. The Pseudomonas aeruginosa strains were isolated and identified from various clinical sample including urine, sputum, pus, wound swab, endo tracheal tube secretions (ETTsec.), blood and cerebrospinal fluid (CSF) etc. The specimens on receipt in the laboratory were inoculated on nutrient agar, blood agar and MacConkey agar. The plates were then incubated at $37^{\circ} \mathrm{C}$ for 24 hours, the growth on above media were then picked up and processed for further identification using standard procedures. P.aeruginosa was identified by colony character with peculiar diffusible pigment production, Gram staining, motility test and biochemical tests like- oxidase test, $\mathrm{O} / \mathrm{F}$ test and growth at $42^{0} \mathrm{C}$. (Govan, 2006) The antibiotic susceptibility test of identified P.aeruginosa strains were performed by modified Kirby Bauer disk diffusion technique (Govan, 2006). The final bacterium inoculation concentration was approximately $10^{8} \mathrm{cfu} / \mathrm{ml}$ that was equal to $0.5 \mathrm{McF}$ arland prepared. Commercially available Muller Hinton Agar with HiMedia discs of using ceftazidime (30mcg), ceftriaxone (30 mcg), cefotaxime (30 mcg), cefepime (30 mcg), gentamicin (10mcg), amikacin (30 mcg), tobramycin (30 mcg), ciprofloxacin $(5 \mathrm{mcg})$, levofloxacin (Le, $5 \mu \mathrm{g}$ ), piperacillin/ tazobactam (100/10mcg), imipenem (10mcg), meropenem $(10 \mathrm{mcg})$, polymyxinB $(300 \mu \mathrm{g})$, colistin (10mcg), norfloxacin (10 $\mathrm{mcg}$ - for urinary isolates). According to CLSI guidelines on Muller Hinton agar plates. (Govan, 2006; Srinivas et al., 2012)

\section{Detection of various phenotypic resistance mechanisms}

ESBL Screening (Clinical and Laboratory Standards Institute, 2016)

Screening of P.aeruginosa for ESBLs production was performed according to the procedures as recommended by the CLSI, using indicator cephalosporins, ceftriaxone $(30 \mu \mathrm{g})$, ceftazidime $(30 \mu \mathrm{g})$, and cefotaxime $(30 \mu \mathrm{g})$. Isolates exhibiting zone size $\leq 25 \mathrm{~mm}$ with ceftriaxone $\leq 22 \mathrm{~mm}$ for ceftazidime and $\leq 27 \mathrm{~mm}$ with cefotaxime were considered as ESBLs producer.

\section{Phenotypic Confirmatory Test for ESBL: (Combined Disc Diffusion Method) (Clinical and Laboratory Standards Institute, 2016)}

A turbidity standard 0.5 McFarland suspension in peptone water was made from the colonies of P.aeruginosa isolate. By using this inoculum, lawn culture was made on Muller Hinton Agar plate. Discs of 
ceftazidime and ceftazidime + clavulanic acid $(30 \mathrm{mcg} / 10 \mathrm{mcg})$ and cephotaxime $(30 \mu \mathrm{g})$ and cephotaxime + clavulanic acid $(30 \mathrm{mcg} / 10$ $\mathrm{mcg}$ ) were placed separately aseptically on the surface of MHA at a distance of $15 \mathrm{~mm}$ apart. Overnight incubation was done at $37^{\circ} \mathrm{C}$. An increase of $\geq 5 \mathrm{~mm}$ in zone diameter of ceftazidime + clavulanic acid and cephotaxime + clavulanic acid in comparison to the zone diameter of ceftazidime and cephotaxime alone confirmed the ESBL production by the organisms.

Methods of Phenotypic Detection of MBL (Clinical and Laboratory Standards Institute, 2016)

Isolates resistant to Imipenem were tested for metallo $\beta$ lactamase production by Imipenem EDTA double disc synergy test (DDST).

\section{EDTA Double Disc Synergy Test (DDST) (Clinical and Laboratory Standards Institute, 2016)}

Lawn culture of the test organism was made onto MHA plates and imipenum disc $(10 \mu \mathrm{g})$ was placed $10 \mathrm{~mm}$ edge to edge from a blank disc contained $10 \mu \mathrm{l}$ of $0.5 \mathrm{M}$ EDTA $(750 \mu \mathrm{g})$. Plates were incubated at $37^{\circ} \mathrm{C}$ overnight. Enhancement of zone of inhibition in the area between imipenem and EDTA disc in comparison with the zone of inhibition on the far side (other side) of the drug is interpreted as a Positive test.

AmpC $\beta$ lactamase detection methods (Clinical and Laboratory Standards Institute, 2016)

Organisms showing resistance to cefoxitin (zone size $<18 \mathrm{~mm}$ ) should be considered as probable AmpC producer and should be confirmed by other methods. ceftazidime $(30 \mu \mathrm{g})$, cefotaxime $(30 \mu \mathrm{g})$ were placed at a distance of $20 \mathrm{~mm}$ from cefoxitin $(30 \mu \mathrm{g})$ on a
MHA plate inoculated with test organism. Isolates showing blunting of zone of inhibition of ceftazidime or cefotaxime adjacent to cefoxitin disc or showing reduced susceptibility to either of the above drugs and cefoxitin are considered as AmpC producer.

\section{Results and Discussion}

In our study, among the 1151 culture positive clinical samples, 100 isolates of P.aeruginosa were isolated $(8.68 \%)$. The predominant sample of isolation was pus/wound swab (17.59\%), followed by ETT Secretion (12.5\%), Ear swab (9.79\%), sputum $(7.66 \%)$ urine (5.74\%), Blood (1.96\%) and CSF (1.02\%) (Table 1).

In our study, among the used $\beta$ lactam other than carbapenems, ceftazidime (61\%), cefepime $(53 \%)$ and fluroquinolones like cipofloxacin (63\%) and levofloxacin(49\%) showed highest resistant. Among the aminoglycosides, gentamicin (41\%) showed highest resistant while tobramicin $(26 \%)$ and amikacin $(28 \%)$ exhibit less resistant.

Among the $\beta$-lactam combination ( $\beta$-lactam combined with $\beta$ latamase inhibitor) by Piperacillin/ tazobactam showed $42 \%$ resistance. The resistant pattern of Aztreonam is $51 \%$. The urine isolates of P.aeruginosa shown $50 \%$ resistant to Norfloxacin. The carbapenems, Imipenem (18\%), Meropenem (19\%), and Doripenem (16\%) showed less resistant. Most of isolates were found to be highly sensitive to Colistin (100\%), Polymyxin B (100\%),

Among 100 strains of P.aeruginosa, which were screened phenotypically for ESBL (33\%), MBL (18\%) and AMP C(0\%), the prevalence of ESBL, MBL and Both ESBL and MBL is $33 \%, 18 \%$, and $9 \%$ respectively. No strain was positive for AMP C (Table 2 and 3). Isolates from ETT. Sec (100\%), Pus 
(48.1\%), Urine $(75.0 \%)$ and wound swab (64.2\%) showed maximum resistant to levofloxacin (Le). Among the combined drug Piperacillin/Tazobactam (25.0\%) shown less resistant.

P.aeruginosa has emerged as a significant pathogen, due to its intrinsic ability to resist many classes of antibiotics as well as its ability to acquire resistance, its virulence, ability to resist killing by various antibiotics and disinfectant, it presents a serious therapeutic challenge for treatment of both community acquired and nosocomial infections. This affects mortility, morbidity and financial implication in therapy of infected patients.

In India, prevalence rate of P.aeruginosa infection varies from $10.5 \%$ to $30 \%$. It ranged from 3 to $16 \%$, in a multicentric study conducted by Ling JM et al., (1995) In other Indian study Pathi et al., reported $8.43 \%$. (Pathi et al.,) The prevalence in our study was found to be $8.68 \%$ which is comparable to above study.

Wound infection and respiratory tract infections were found to be commonly affected by P.aerugiosa. In this study the predominant sample of isolation was pus/wound swab (17.59\%), followed by ETT secretion (12.5\%), ear swab (9.79\%), sputum (7.66\%) urine $(5.74 \%)$, Blood $(1.96 \%)$ and CSF (1.02\%). S. Senthamarai et al., (47.11\%) (Senthamarai et al., 2014) and Vijaya Chaudhari et al., (35.3\%) also reported highest rate of isolation in pus. (Vijaya Chaudhari et al., 2013)

In a study conducted in Punjab, India, Arora et al., found highest recovery rates were from urine $(36 \%)$, followed by wound discharge (20\%), tracheal aspirate (8\%), ear discharge (5\%) and sputum (4\%). (Arora et al., 2011)

Another study by Javiya et al., from Gujarat, India, reported higher isolation rates from urine, pus and sputum which accounts to $27 \%$ each, followed by ET secretion 14\%. (Javiya et al., 2008) This variation among these studies could be due to the difference in study period and sample size, geographical location and patient population.

Fig.1

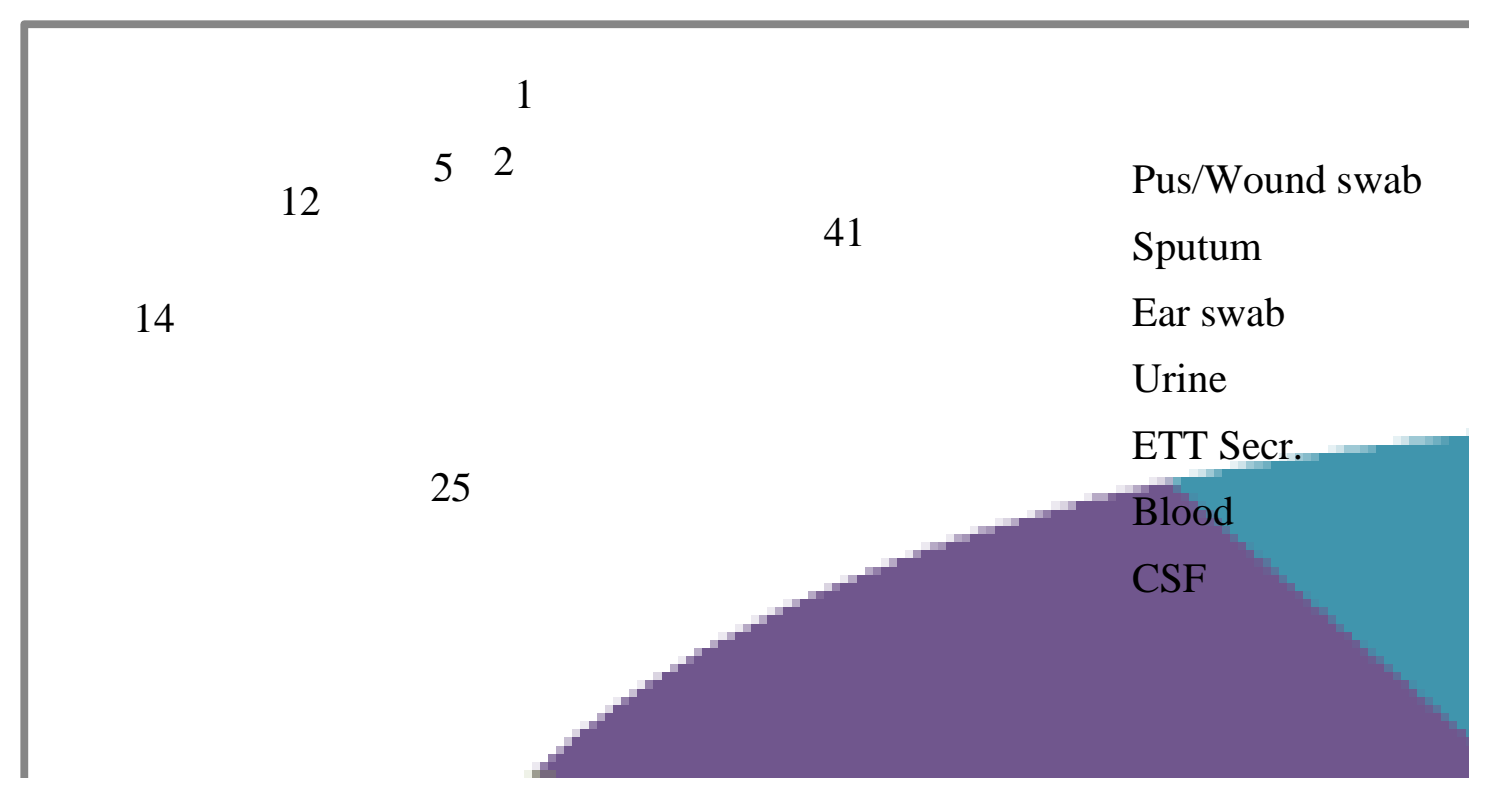


Table.1 Isolation rate of $P$. aeruginosa from different clinical Sample $(\mathrm{N}=1151)$

\begin{tabular}{|l|}
\hline Sample \\
\hline Pus/ Wound swab \\
\hline Sputum \\
\hline Ear Swab \\
\hline Urine \\
\hline ET T Sec. \\
\hline Blood \\
\hline CSF \\
\hline TOTAL \\
\hline
\end{tabular}

\begin{tabular}{|c|c|}
\hline $\mathbf{N}$ & $\mathbf{N o}(\%)$ \\
\hline 233 & $41(17.59)$ \\
\hline 326 & $25(7.66)$ \\
\hline 143 & $14(9.79)$ \\
\hline 209 & $12(5.74)$ \\
\hline 40 & $5(12.5)$ \\
\hline 102 & $2(1.96)$ \\
\hline 98 & $1(1.02)$ \\
\hline 1151 & $100(100)$ \\
\hline
\end{tabular}

Table.2 Antibiotic susceptibility pattern of $P$. aeruginosa in different clinical specimen

\begin{tabular}{|l|}
\multicolumn{1}{|c|}{ ANTIBIOTICS } \\
\hline Ceftriaxone (30) \\
\hline Ceftazidime (30) \\
\hline Cefipime \\
\hline Piperacillin-Tazobactam \\
\hline Gentamicin \\
\hline Amikacin \\
\hline Tobramycin \\
\hline Ciprofloxacin \\
\hline levofloxacin \\
\hline Imipenem \\
\hline Meropenem \\
\hline Colistin \\
\hline Polymyxin b \\
\hline Aztreonam \\
\hline Norfloxacin \\
\hline
\end{tabular}

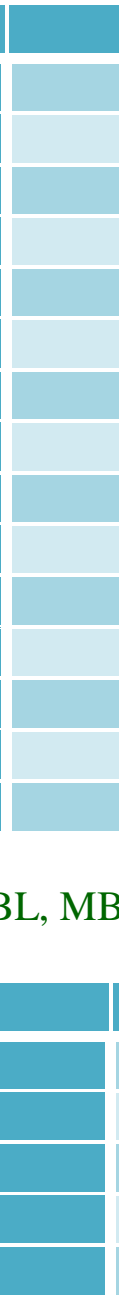

\section{SENSITIVE}

28

39

47

58

59

72

74

37

51

82

81

100

100

49

48

\section{RESISTANT}

\begin{tabular}{l}
62 \\
61 \\
53 \\
42 \\
41 \\
28 \\
26 \\
63 \\
49 \\
18 \\
19 \\
00 \\
00 \\
51 \\
52 \\
\hline
\end{tabular}

Table.3 Prevalence of ESBL, MBL, Amp c and from different clinical isolates ( $n=100)$

\begin{tabular}{|l|c|c|}
\hline N=100 & No of isolates & Percentage \\
\hline MIDR & 28 & 28 \\
\hline ESBL & 33 & 33 \\
\hline MIBL & 18 & 18 \\
\hline BOTH ESBL AND MIBL & 09 & 9 \\
\hline AMIP C & 0 & 0 \\
\hline
\end{tabular}

Most of isolates were found to be highly sensitive to colistin (100\%), polymyxin B (100\%), doripenem (89.0\%) imipenem (84 $\%$ ), amikacin $(76.0 \%)$ and piperacillin + tazobactum (75\%). As the bacterial strains that show resistance to three or more categories of antibiotics are defined as multidrug resistant (MDR) strains, 
(Senthamarai et al., 2014) MDR strains of P.aeruginosa isolated in this study were $28 \%$.

In our study P.aeruginosa showed highest resistant to $\beta$-lactum antibiotics and fluroquinolones. Among the $\beta$ lactam drugs, ceftazidime (61\%) and cefepime (53\%) showed the highest resistance in this present study. K.M Mohanasundaram et al., (84.6\%), (Mohanasundaram, 2011) Yapar et al., (84\%) (Ayse Yüce et al., 2009) and Ibukun et al., (79.4\%), (Ibukun et al., 2007) reported more resistance against ceftazidime in their study. Our study is in line with the reports of Diwivedi et al., (63\%) (Diwivedi et al., 2009) \& Arya et al., (55.4\%). (Arya et al., 2005) The reason for high resistance of third and fourth generation cephalosporin may be due to indiscriminate use of third and fourth generation cephalosporin as broad spectrum empirical therapy and the secretion of ESBL enzymes mediate the resistance by hydrolysis of $\beta$-lactam ring of $\beta$-lactam antibiotics. Other mechanisms of drug resistance to $\beta$-lactam group of antibiotics in Pseudomonas aeruginosa are due to loss of outer membrane protein, production of class $\mathrm{C} A \mathrm{Amp} \beta-$ lactamase and altered target sites.

Our study showed $33(33 \%)$ isolates were ESBL producer. $42.30 \%$ ESBL producer were observed in the study of (Varun Goel et al., 2013) Lower ESBL producer were seen in the studies by (Prashant et al., 2011) and Agarwal et al., which were $22.22 \%$ \& $20.27 \%$ respectively (Aggarwal et al., 2008)

The ESBL enzymes are inhibited by $\beta$ lactamase inhibitors, viz., clavulanic acid. Hence the use of $\beta$-lactam $/ \beta$-lactamase inhibitor combination may be an alternative to 3rd generation cephalosporin, but the effect of this combination varies depending on the subtype of ESBL present. In our study $\beta$ lactamase inhibitor resistance was ranged from $42 \%$ to $57 \%$. Similar resistance also observed by Senthamarai et al., (37.5\% to $56.73 \%$ ) (Senthamarai et al., 2014) and K.M Mohanasundaram et al., (40.3\%). (Mohanasundaram, 2011) In therapeutic part, increasing resistance to $\beta$ lactam inhibitors is a major problem which makes them less reliable for therapeutic purposes. Though imipenem was found unaffected by the action of the enzymes in many studies, MBL production in our study was $(18 \%)$ which is comparable with the studies of Ibukun et al., and Senthamarai et al., (15.38\%). (Senthamarai et al., 2014; Ibukun et al., 2007), (Prashant et al., 2011; Agarwal et al., 2008; Jayakumar and Appalraju, 2007; Navneeth et al., 2002) and slightly raised level of carbapenem resistance were reported by Variya et al., (25\%). (Variya et al., 2008) The percentage variation in the resistance mechanism could be due to the study environment where the study was done. These carbapenem agents may be of benefit in the treatment of ESBL infection; however, indiscriminate use of these agents may promote increased resistance to carbapenems. None of our isolates showed AmpC $\beta$ lactamase.

P.aeruginosa showed higher resistance to many other classes of antibiotics, including fluoroquinolones $(49 \%$ to $63 \%)$ and aminoglycosides (26\% to $46 \%$ ). This is due to the coexistence of genes encoding drug resistance to other antibiotics on the plasmids which encode ESBL. This fact has also been observed in our study. Among the aminoglycoside group, gentamycin showed highest resistance (41\%). Minimal resistance was observed with other aminoglycoside such as tobramycin (26\%) and amikacin (28\%) which is shows promising effect in treatment. Ciprofloxacin showed (63\%) $61.53 \%$ resistance to P.aeruginosa in our study. In various reports on ciprofloxacin resistance to P.aeruginosa was ranged between 0-89\% (Algun et al., 2004). 
It is evident from the study that nowadays P.aeruginosa is becoming resistant to cephalosporins, aminoglycosides and even beta lactam $(\mathrm{BL})$ - beta lactamase inhibitor (BLI) combinations and Carbapenems. Furthermore, infections with such strains may result in poor or untoward clinical outcomes that may increase morbidity, mortality and economic burden. Proper use of antibiotics following a proper antibiotic policy is the best way to control spreading of this superbug. To prevent the spread of the resistant bacteria it is critically important to have strict antibiotic policies. To minimize the resistance to in use routine antibiotics, it is desirable that the antibiotic susceptibility pattern of bacterial pathogens like P.aeruginosa in clinical units should be continuously monitored. As there are few studies available in our locality, studies like this would help to formulate the antibiotic guidelines to the physician in treatment part which in turn has a great impact in preventing the mortality and morbidity associated with Pseudomonas aeruginosa infections.

\section{References}

Aggarwal R, Chaudhary U, Bala K. Detection of extended-spectrum beta-lactamase in Pseudomonas aeruginosa. Indian $J$ Pathol Microbiol. 2008; 51: 222-4.

Algun A, Arisoy, Gunduz T, Ozbakkaloglu B. the resistance of Pseudomonas aeruginosa strains to fluoroquinolones group of antibiotics. Ind J Med Micro. 2004; 22(2); 112-14.

Arora D, Jindal N, Romit RK. Emerging antibiotic resistance in Pseudomonas: A Challenge. International Journal of Pharmacy and Pharmaceutical Science. 2011; 3(2):82-84.

Arya M, Arya P, Biswas D, Prasad R. The antimicrobial susceptibility pattern of the bacterial isolates from post- operative wound infections. Indian $J$ Pathol Microbiol. 2005; 48(2): 266-69.

Ayse Yüce, Nur Yapar, Oya Eren Kutsoylu. Evaluation of antibiotic resistance patterns of pseudomonas aeruginosa and Acinetobacter spp. strains isolated from intensive care patients between 20002002 and 2003-2006 periods in Dokuz Eylul University Hospital, Izmir Mikrobiyol Bul. 2009; 43(2):195-202.

Clinical and Laboratory Standards Institute. Performance Standards for Antimicrobial Susceptibility Testing; $26^{\text {th }}$ Informational Supplement (M100-S21). Wayne, PA: Clinical and Laboratory Standards Institute; 2016.

Diwivedi M, Mishra A, Singh RK, Azim A, Baronia AK, Prasad KN. The nosocomial cross - transmission of Pseudomonas aeruginosa between patients in a tertiary intensive care unit. Indian J Pathol Microbiol. 2009; 52(4): 509-13.

$\mathrm{Du}$ Bois V, Arpin C, Melon $\mathrm{M}$ et al., Nosocomial outbreak due to a multiresistance strain of Pseudomonas aeruginosa P12: efficacy of cefepimeamikacin therapy and analysis of $\beta$ lactam resistance. J. Clin. Microbiol. 2001; 39: 2072-2078.

Gales AC, Jones RN, Turnidge J, Rennie, R Ramphal R. Characterisation of Pseudomonas aeruginosa isolates: occurrence, rate antimicrobial susceptibility pattern and molecular typing in the Global Sentry antimicrobial surveillance program 1997-1999. Clin. Infect. Dis. 2001; 32: $146-155$.

Govan J. R. W. Pseudomonads and nonfermenters. In Medical microbiology A Guide to Microbial Infections: Pathogenesis, Immunity, Laboratory Diagnosis and Control. Eds. Greenwood David, Slack Richard C.B, Peutherer 
John F. 6th edn. Edinburg: Churchill Livingstone; 2008; p. 282-287.

Govan, J. R. W. Pseudomonas, Stenotrophomonas, Burkholderia. In: Mackie and Mc Cartney Practical Medical Microbiology. Eds. Collee JG, Fraser AG, Marmion BP \& Simmons A. 14th ed. Edinburg: Churchill Livingstone; 2006; p 413-424.

Hernandez J, Ferrs MA, Hernandez M, Owen RJ. Arbitrary primed PCR fingerprint and serotyping of clinical Pseudomonas aeruginosa strains. FEMS Immunology and Medical Microbiology. 1997: (17); $37-47$.

Ibukun A, Tochukwu N, Tolu O. Occurrence of ESBL and MBL in clinical isolates of Pseudomonas aeruginosa From Lagos, Nigeria. Journal of American Science. 2007; 3(4): 81-85.

Javiya VA, Ghatak SB, Patel KR, Patel JA. Antibiotic susceptibility patterns of Pseudomonas aeruginosa at a tertiary care hospital in Gujarat, India. Indian J Pharmacol. 2008; 40(5):230-4.

Jaykumar S, Appalraju B. The prevalence of multi and pan drug resistant Psuedomonas aeruginosa with respect to ESBL and MBL in a tertiary care hospital. Indian $J$ Pathol Microbiol. 2007; 50 (4): 922-25.

Ling J M, Cheng AF. Antimicrobial resistance of clinical isolates from 1987 to 1993 in Hong Kong. HKMJ. 1995; 1(3):212-18.

Mohanasundaram KM. The antimicrobial resistance pattern in the clinical isolates of Pseudomonas aeruginosa in a tertiary care hospital: 2008-2010(a 3 year study). Journal of Clinical and Diagnostic Research. 2011, Vol-5(3); 491-94.

Navneeth BV, Sridaran D, Sahay D, Belwadi MA preliminary study on the metallo betalactamase producing Pseudomonas aeruginosa in hospitalised patients. Indian J Med Res. 2002; 112: 264-67.

Pathi B, Mishra SN, Panigrahi K, Poddar N, Lenka PR, Mallick B, Pattanik D, Jena J. Prevalence and antibiogram pattern of Pseudomonas aeruginosa in a tertiary care hospital from Odisha, India. Transworld Medical Journal. 1(3):7780.

Prashant Durwas Peshattiwar, Basavaraj Virupaksappa Peerapur. ESBL and MBL mediated resistance in Pseudomonas aeruginosa: an emerging threat to clinical therapeutics. Journal of Clinical and Diagnostic Research. 2011, Vol-5(8); 1552-554.

Senthamarai S, Suneel Kumar Reddy A, and Sivasankari S et al., Resistance Pattern of Pseudomonas aeruginosa in a Tertiary Care Hospital of Kanchipuram, Tamilnadu, Indian Journal of Clinical and Diagnostic Research. 2014 May, Vol-8(5): 30-32.

Srinivas B, Lalitha Devi D, Narasinga Rao B. A prospective study of Pseudomonas aeruginosa and its antibiogram in a teaching hospital of Rural setup. Journal of Pharmaceutical and Biomedical sciences. 2012; 22:23-29.

Syed Arshi, Thakur Manzoor, Shafiq Syed, Mr. Sheikh Ullah Assad. In-vitro sensitivity patterns of Pseudomonas aeruginosa strains isolated from patients at skims - role of antimocribials in the emergence of multiple resistant strains. JKPractitioner. 2007; 14(1):31-34.

Variya A, Kulkarni N, Kulkarni M, et al., The incidence of metallo beta lactamase producing Pseudomonas aeruginosa among ICU patients. Indian J Med Res. 2008; 127: 398-402.

Varun Goel, Sumati A. Hogade, SG Karadesai. Prevalence of extendedspectrum beta-lactamases, AmpC beta-lactamase, and 
metallo-beta-lactamase producing Pseudomonas aeruginosa and Acinetobacter baumannii in an intensive care unit in a tertiary Care Hospital Journal of the Scientific Society. 2013 40(1), pp. 28-31.
Vijaya Chaudhari, Sandeep Gunjal, Mukesh Mehta. Antibiotic resistance patterns of Pseudomonas aeruginosa in a tertiary care hospital, in Central India. International Journal of Medical science and Public Health. 2013; Vol 2(2) 38689.

\section{How to cite this article:}

Trinain Kumar Chakraverti and Purti C. Tripathi. 2018. Prevalence and Antibiotic Resistant Pattern of Pseudomonas aeruginosa at a Tertiary Care Centre of North India. Int.J.Curr.Microbiol.App.Sci. 7(09): 1061-1069. doi: https://doi.org/10.20546/ijcmas.2018.709.126 